\title{
The Limiting Absorption and Amplitude Principles for the Diffraction Problem with Two Unbounded Media
}

\author{
D. Eidus
}

School of Mathematical Sciences, Tel-Aviv University, Tel-Aviv, Israel

\begin{abstract}
We consider the classical diffraction problem for the wave propagation in the case where the propagation speed is piecewise constant, and the surface separating two media is unbounded. The validity of the limiting absorption and amplitude principles is proved.
\end{abstract}

\section{Introduction}

We deal with the asymptotic behaviour (as $t \rightarrow+\infty$ ) of the following Cauchy problem

$$
\begin{aligned}
\mu(x) w_{t t}-\Delta w & =e^{-i \omega t} f(x), \\
\left.w\right|_{t=0} & =\left.w_{t}\right|_{t=0}=0,
\end{aligned}
$$

in the space $\mathbb{R}^{n}=\{x\}, x=\left(x_{1}, \ldots, x_{n}\right)$ (we denote the radius vector of the point $x$ by the same letter), where $n \geqq 3, \omega=$ const $>0, f$ belongs to some $L^{2}$-weighted space,

$$
\mu(x)=\frac{1}{a^{2}(x)} .
$$

$a(x)$ is a wave propagation speed.

We assume that $\mu(x)$ has only two values:

$$
\mu(x)=\mu_{j} \quad \text { if } x \in \Omega_{j}, \quad j=1,2
$$

where $\mu_{j}=$ const $>0$,

$$
\begin{array}{ll}
\Omega_{1}=\left\{x: x \in \mathbb{R}^{n},\right. & \left.x_{n}>\varphi(\tilde{x})\right\}, \\
\Omega_{2}=\left\{x: x \in \mathbb{R}^{n},\right. & \left.x_{n}<\varphi(\tilde{x})\right\},
\end{array}
$$

where $\tilde{x}=\left(x_{1}, \ldots, x_{n-1}\right) \in \mathbb{R}^{n-1}, \varphi(\tilde{x})$ is some given function, defined on $\mathbb{R}^{n-1}$ and satisfying the condition

$$
\varphi \in C^{1}\left(\mathbb{R}^{n-1} \backslash\{0\}\right)
$$


Let $S$ be the media separating surface

$$
x_{n}=\varphi(\tilde{x}),
$$

$v=\left(v_{1}, \ldots, v_{n}\right)$ be the unit normal at the point $x \in S$, where

$$
v_{n}>0 \text {. }
$$

$\langle\cdot, \cdot\rangle$ be the scalar product in the complex $n$-dimensional space $\mathbb{C}^{n}$. We shall impose the following conditions on $S$ : for any $x \in S \backslash\{0\}$,

$$
\begin{gathered}
v_{n} \geqq c_{1}>0 . \\
|\langle x, v\rangle| \leqq c_{2},
\end{gathered}
$$

where $c_{j}, j=1,2$, do not depend on $x \in S \backslash\{0\}$.

Let us assume the continuity of $w$ and $\partial w / \partial v$ on $S$.

Note 1.1. The following condition implies (i), (ii): if $|\tilde{x}| \rightarrow \infty$, then

$$
\begin{aligned}
\varphi(\tilde{x}) & =Q(\tilde{x})+O(1) \\
\nabla \varphi(\tilde{x}) & =\nabla Q(\tilde{x})+O\left(|x|^{-1}\right),
\end{aligned}
$$

where

$$
\begin{gathered}
Q(\tilde{x}) \in C\left(\mathbb{R}^{n-1}\right) \cap C^{1}\left(\mathbb{R}^{n-1} \backslash\{0\}\right), \\
Q(k x)=k Q(\tilde{x}), \quad \forall k>0, \quad \tilde{x} \in \mathbb{R}^{n-1} .
\end{gathered}
$$

In the case $\varphi=Q, S$ is a smooth cone with the vertex $x=0$. Let us notice that our problem is not trivial in this case either, because it is impossible to separate space variables when $\mu_{1} \neq \mu_{2}$.

Introduce the functional spaces $L_{\beta}^{2}, H_{\beta}^{m}$ with the norms

$$
\begin{aligned}
\|\cdot\|_{\beta}^{2} & =\int(1+|x|)^{\beta}|\cdot|^{2} d x, \\
\|\cdot\|_{\beta, m}^{2} & =\int(1+|x|)^{\beta} \sum_{0 \leqq|\tau| \leqq m}\left|D^{\tau}\right|^{2} d x,
\end{aligned}
$$

where we integrate over the whole $\mathbb{R}^{n}$ and $\tau$ is a multiindex (i.e., $\tau=\left(\tau_{1}, \ldots, \tau_{n}\right), \tau_{j}$ are non-negative integers),

$$
\begin{aligned}
& |\tau|=\sum_{j=1}^{n} \tau_{j}, \\
& D^{\tau}=\frac{\partial|\tau|}{\partial x_{1}^{\tau_{1}} \cdots \partial x_{n}^{\tau_{n}}}
\end{aligned}
$$

is a distribution derivative, $\beta \in \mathbb{R}$.

In this paper, we prove the validity of the limiting absorption principle (LABP) for the operator

$$
A=-\mu^{-1} \Delta
$$

corresponding to the problem (1.1), (1.2), and the validity of the limiting amplitude principle (LAP) for the problem (1.1), (1.2). The last statement means that the 
following asymptotic formula

$$
w=e^{-i \omega t} u+o(1)
$$

holds is $L_{-\beta}^{2}$ as $t \rightarrow+\infty$, where $\beta=3$ for $n>3$ and $\beta>3$ for $n=3$, $u$ is the solution of the equation,

$$
\Delta u+\omega^{2} \mu u+f=0
$$

given by LABP:

$$
u=\lim _{z \rightarrow \omega^{2}+o i} R_{z}\left(\mu^{-1} f\right) .
$$

Here $R_{z}$ is the resolvent of the operator (1.13) treated in the space $L^{2}$ with the weight $\mu$, and the convergence in (1.16) takes place in $L_{-2}^{2}$. The proof of LAP is based on the following theorem (see [2]):

Theorem 1.1. Let $H$ be a Hilbert space, $A$ be a linear self-adjoint operator in $H$, $(A u, u)>0$ if $u \neq 0, R_{z}$ be the resolvent of $A$. Let $H$ be continuously embedded in a Banach space $B$, and the following conditions be satisfied:

i) For some $f \in H$ the limits

$$
\lim _{z \rightarrow \lambda \pm o l} R_{z} f=u_{\lambda}^{ \pm}
$$

exist in $B$ for any $\lambda>0$.

ii) The function

$$
\Theta(\lambda)=u_{\lambda}^{+}-u_{\lambda}^{-}
$$

with values in $B$ satisfies the Hölder condition in any interval $a \leqq \lambda \leqq b, a>0$.

iii) In some interval $0<\lambda \leqq b$

$$
\|\Theta(\lambda)\|_{B} \leqq C \lambda^{-\tau},
$$

where $c, \tau$ are constant, $0 \leqq \tau<1 / 2$.

Let $w$ be the solution of the Cauchy problem in $H$ :

$$
\begin{gathered}
\frac{d^{2} w}{d t^{2}}+A w=e^{-i \omega t} f, \quad t>0, \\
\left.w\right|_{t=0}=\left.\frac{d w}{d t}\right|_{t=0}=0 .
\end{gathered}
$$

Then for each $\omega=$ const $>0$ in $B$

$$
\lim _{t \rightarrow+\infty}\left(w-e^{-i \omega t} u_{\omega^{2}}^{+}\right)=0 .
$$

Note 1.2. The well known radiation principle, which coincides with the Sommerfeld principle when $\mu_{1}=\mu_{2}$, is connected generally with LABP and LAP (see $[1,3])$. But we did not succeed in finding a radiation condition for our operator $A$. There is a large literature about these three principles. (See for instance $[1,3,4,5]$.) 


\section{The Operator of the Problem and the Main a priori Estimates}

Let us introduce the Hilbert space $L^{2}(\mu)$ of functions, defined on $\mathbb{R}^{n}$, with the scalar product

$$
(u, v)=\int \mu u \bar{v} d x
$$

and the corresponding norm $\|\cdot\|$. It is obvious that the norms $\|\cdot\|$ and $\|\cdot\|_{0}$ are equivalent. Let us define the operator $A$ in $L^{2}(\mu)$ by the equality (1.13) on the domain

$$
D(A)=H_{0}^{2} \text {. }
$$

The condition (2.2) generalizes the classical condition of the continuity of the solution and its normal derivative on $S$. It is well known that $A$ is positive and selfadjoint in $L^{2}(\mu)$.

Now we shall prove that the resolvent $R_{z}$ of $A$, where

$$
z=\lambda+\varepsilon i, \quad \varepsilon \neq 0
$$

treated as follows

$$
R_{z}: L_{\beta}^{2} \rightarrow L_{-\beta}^{2}, \quad \beta>0,
$$

where $\beta$ is sufficiently large, is uniformly bounded near the real axis (i.e. $\left\|R_{z}\right\|$ is bounded).

Let $f \in L_{0}^{2}, z$ be defined by (2.3),

$$
u_{z}=R_{z} f
$$

Then a.e. on $\mathbb{R}^{n}$

$$
\Delta u_{z}+z \mu u_{z}+\mu f=0
$$

Hence

$$
\begin{aligned}
\int\left(\lambda \mu\left|u_{z}\right|^{2}-\left|\nabla u_{z}\right|^{2}\right) d x & =-\operatorname{Re}\left(f, u_{z}\right), \\
\varepsilon\left\|u_{z}\right\|^{2} & =-\operatorname{lm}\left(f, u_{z}\right) .
\end{aligned}
$$

Since (2.7), (2.8), then

$$
\varepsilon\left\|u_{z}\right\|_{0,1}^{2} \leqq c(1+|\lambda|+|\varepsilon|)\left(|f|,\left|u_{z}\right|\right)_{0},
$$

where $(\cdot, \cdot)_{0}$ is the scalar product in $L_{0}^{2}$ and

$$
C=\max \left(\mu_{1}, \mu_{2}\right) \text {. }
$$

Lemma 2.1. Let $f \in L_{0}^{2}, \beta>3$ for $n=3, \beta=3$ for $n>3$,

$$
\begin{gathered}
0<|\varepsilon| \leqq 1 \\
-1 \leqq \lambda \leqq M
\end{gathered}
$$

(see (2.3)), where $M$ is some constant, and the conditions (1.4), (1.7) be satisfied. Then

$$
\left\|u_{z}\right\|_{-\beta, 1}^{2} \leqq c\left(|f|,\left|u_{z}\right|+\left|\nabla u_{z}\right|\right)_{0} .
$$

where $c$ does not depend on $f, \lambda, \varepsilon$ (but may depend on $M, \beta$ ). 
Proof. Multiplying (2.6) by $\partial_{n} \bar{u}$, where $\partial_{n} \Psi=\partial \Psi / \partial x_{n}$, and integrating over $\mathbb{R}^{n}$ we obtain

$$
\lambda\left(\mu_{2}-\mu_{1}\right) / 2 \int_{S}\left|u_{z}\right|^{2} v_{n} d x-\varepsilon \operatorname{Im}\left(u_{z}, \partial_{n} u_{z}\right)=\left(f, \partial_{n} u_{z}\right)
$$

Since (1.7), (2.9), (2.13) then

$$
|\lambda| \cdot\left|u_{2}-\mu_{1}\right| \int_{S}\left|u_{z}\right|^{2} d x \leqq c\left(|f|,\left|u_{z}\right|+\left|\partial_{n} u_{z}\right|\right)_{0}
$$

Here and below $c$ does not depend on $f, \varepsilon, \lambda$. First, let us consider the case where

$$
f \in C^{1}\left(\mathbb{R}^{n}\right) \text {. }
$$

Then, $u \in C^{1}\left(\Omega_{j}\right), j=1,2$. Let $x_{0} \in \mathbb{R}^{n} \backslash S, x \in \mathbb{R}^{n}$,

$$
r=x-x_{0} \text {. }
$$

Multiply (2.6) by $\partial_{r} \bar{u}_{z}$, where $x_{0}$ is fixed,

$$
\partial_{r} \Psi=\frac{\partial \Psi}{\partial r}
$$

and integrate over $\mathbb{R}^{n}$ with respect to $x$. Then for $x_{0} \in \Omega_{j}, j=1,2$,

$$
\begin{aligned}
& (-1)^{j} \lambda\left(\mu_{2}-\mu_{1}\right) / 2 \int_{S}\left|u_{z}\right|^{2}\left\langle e_{0}, v\right\rangle d x+(n-3) / 2 \int|r|^{-1}\left(\left|\nabla u_{z}\right|^{2}-\lambda \mu\left|u_{z}\right|^{2}\right) d x \\
& +\int|r|^{-1}\left(\left|\partial_{r} u_{z}\right|^{2}-\lambda \mu\left|u_{z}\right|^{2}\right) d x=\varepsilon \operatorname{Im}\left(u_{z}, \partial_{r} u_{z}\right)-\operatorname{Re}\left(f, \partial_{r} u_{z}\right) .
\end{aligned}
$$

Now we multiply (2.6) by $|r|^{-1} u_{z}$ and integrate over $\mathbb{R}^{n}$. After simple calculations, we obtain

$$
\int|r|^{-1}\left(\left|\nabla u_{z}\right|^{2}-\lambda \mu\left|u_{z}\right|^{2}\right) d x=U+\operatorname{Re}\left(|r|^{-1} f, u_{z}\right),
$$

where $U=-2 \pi\left|u_{z}\left(x_{0}\right)\right|^{2}$ if $n=3, U=-(n-3) / 2 \int|r|^{-3}\left|u_{z}\right|^{2} d x$ if $n>3$. For $n=3$ we obtain from (2.9), (2.14), (2.16) and (2.17) as follows:

$$
\left|u_{z}\left(x_{0}\right)\right|^{2} \leqq C_{1}\left(|f|,\left|u_{z}\right|+\nabla u_{z} \mid\right)_{0}+C_{2}\left(|r|^{-1}|f|,\left|u_{z}\right|\right)_{0} .
$$

Let us multiply (2.18) by $\left(1+\left|x_{0}\right|\right)^{-\beta}, \beta>3$, and integrate over $\mathbb{R}^{3}$ with respect to $x_{0}$. Then we obtain

$$
\left\|u_{z}\right\|_{-\beta}^{2} \leqq C\left(|f|,\left|u_{z}\right|+\left|\nabla u_{z}\right|\right)_{0}
$$

Since (2.6), (2.19), then

$$
\left\|\nabla u_{z}\right\|_{-\beta}^{2} \leqq C\left(|f|,\left|u_{z}\right|+\left|\nabla u_{z}\right|\right)_{0} .
$$

Now (2.12) follows immediately from (2.19), (2.20) when (2.15) takes place. But the set $L_{0}^{2} \cap C^{1}\left(\mathbb{R}^{n}\right)$ is dense in $L_{0}^{2}$, therefore (2.12) is valid for any $f \in L_{0}^{2}$.

In the case $n>3$ we obtain from (2.9), (2.14), (2.16), (2.17) in the same way as above:

$$
\int|r|^{-3}\left|u_{z}\right|^{2} d x \leqq C_{1}\left(|f|,\left|u_{z}\right|+\left|\nabla u_{z}\right|\right)_{0}+C_{2}\left(|r|^{-1}|f|,\left|u_{z}\right|\right)_{0}
$$


Multiply (2.21) by $\left(1+\left|x_{0}\right|\right)^{-\beta_{0}}, \beta_{0}>n$, and integrate over $\mathbb{R}^{n}$ with respect to $x_{0}$. Using the inequality

$$
\frac{C^{\prime \prime}}{(1+|x|)^{\gamma}} \leqq \int \frac{d x_{0}}{\left(1+\left|x_{0}\right|\right)^{\beta_{0}}\left|x-x_{0}\right|^{\gamma}} \leqq \frac{C^{\prime}}{(1+|x|)^{\gamma}}
$$

where $0 \leqq \gamma<n$ and $C^{\prime}, C^{\prime \prime}$ do not depend on $x \in \mathbb{R}^{n}$, we obtain

$$
\left\|u_{z}\right\|_{-3} \leqq C\left(|f|,\left|u_{z}\right|+\left|\nabla u_{z}\right|\right)_{0}
$$

As above (2.12), where $\beta=3$, follows from (2.23). The proof is complete.

Theorem 2.1. Let the conditions (1.4), (1.7) be satisfied, $\varepsilon \neq 0, \lambda \leqq M$, where $M$ is some constant, $f \in L_{\beta}^{2}, \beta=3$ if $n>3, \beta>3$ if $n=3$. Then

$$
\left\|u_{z}\right\|_{-\beta, 1}^{2} \leqq C\|f\|_{\beta},
$$

where $C$ does not depend on $f, \varepsilon, \lambda$.

Proof. If (2.10), (2.11) are valid, then (2.24) follows from (2.12). If $\lambda \leqq-1$ or $|\varepsilon|>1$, then (2.24) follows immediately from (2.7), (2.8). Thus (2.24) is proved.

Corollary 2.1. Let the conditions (1.4), (1.7) be satisfied. Then the spectrum of the operator $A$ is absolutely continuous.

Corollary 2.2. Let the conditions of Theorem 2.1 be satisfied. Then the following inequality holds:

$$
\left\|u_{z}\right\|_{-\beta, 2} \leqq C\|f\|_{\beta} .
$$

In fact (2.25) follows immediately from (2.6), (2.24) and the well known inequality (see for instance [1])

$$
\|g\|_{-\beta, 2} \leqq C\left(\|\Delta g\|_{-\beta}+\|g\|_{-\beta, 1}\right) .
$$

Corollary 2.3. Let the conditions (1.4), (1.7) be satisfied, and $w$ be the solution of the following problem:

$$
\begin{aligned}
\frac{d^{2} w}{d t^{2}}+A w & =0, \\
\left.w\right|_{t=0} & =f_{1}, \\
\left.\frac{d w}{d t}\right|_{t=0} & =f_{2},
\end{aligned}
$$

where $f_{1} \in D(A) \cap L_{\beta}^{2}, f_{2} \in D(\sqrt{A}) \cap L_{\beta}^{2}, \beta$ as above. Then $\|w\|_{-\beta, 2} \rightarrow 0$ as $t \rightarrow \infty$.

This result follows from (2.25) and from the corresponding considerations of [1]. Lemma 2.2. Let $\xi_{j}(x) \geqq 0, j=\leqq 1,2$ be Lebesgue measurable functions on $\mathbb{R}^{n}, \alpha_{1}<$ $\alpha_{2}<\alpha_{3}$. Then

$$
\int \xi_{1}^{\alpha_{2}} \xi_{2} d x \leqq\left(\int \xi_{1}^{\alpha_{1}} \xi_{2} d x\right)^{1 / p}\left(\int \xi_{1}^{\alpha_{3}} \xi_{2} d x\right)^{1 / q}
$$

where

$$
p=\frac{\alpha_{3}-\alpha_{1}}{\alpha_{3}-\alpha_{2}}, \quad q=\frac{\alpha_{3}-\alpha_{1}}{\alpha_{2}-\alpha_{1}} .
$$


The inequality (2.30) is a special case of the Hölder inequality with $p, q$ as above.

Corollary 2.4. Let $\alpha_{1}<\alpha_{2}<\alpha_{3}, v \in H_{\alpha_{3}}^{m}, m \geqq 0$ be an integer. Then

$$
\|v\|_{\alpha_{2}, m} \leqq\|v\|_{\alpha_{1}, m}^{1 / p}\|v\|_{\alpha_{3}, m}^{1 / q}
$$

where $p, q$ as above.

Lemma 2.3. Let (1.4), (1.7), (2.10), (2.11) be satisfied, $f \in L_{0}^{2}, 0<\alpha<3$. Then

$$
\left\|u_{z}\right\|_{-\alpha, 1}^{2} \leqq C \varepsilon^{-(1-\alpha / \beta)}\left(|f|, u_{z}|+| \nabla u_{z} \mid\right)_{0},
$$

where $\beta$ as in Theorem 2.1, $C$ does not depend on $f, \lambda, \varepsilon$.

Proof. Because of (2.9), (2.10), (2.11)

$$
\left\|u_{z}\right\|_{0,1}^{2} \leqq C \varepsilon^{-1}\left(|f|,\left|u_{z}\right|+\left|\nabla u_{z}\right|\right)_{0} .
$$

Since (2.12) and (2.31), where $v=u_{z}, \alpha_{1}=-\beta, \alpha_{2}=-\alpha, \alpha_{3}=0, m=1$, we have

$$
\left\|u_{z}\right\|_{-\alpha, 1} \leqq\left\|u_{z}\right\|_{-\beta}^{\alpha / \beta}\left\|u_{z}\right\|_{0,1}^{1-\alpha / \beta} \leqq C \varepsilon^{-1 / 2(1-\alpha / \beta)}\left(|f|,\left|u_{z}\right|+\nabla u_{z} \mid\right)_{0}^{1 / 2}
$$

and (2.32) is proved.

Corollary 2.5. Let the conditions (1.4), (1.7), (2.10), (2.11), $0<\alpha<3, f \in L_{\alpha}^{2}$ be satisfied. Then

$$
\left\|u_{z}\right\|_{-\alpha, 1} \leqq C \varepsilon^{-(1-\alpha / \beta)}\|f\|_{\alpha} .
$$

Indeed (2.34) follows from (2.33) and

$$
\left(|f|,\left|u_{z}\right|+\left|\nabla u_{z}\right|\right)_{0} \leqq C\left\|u_{z}\right\|_{-\alpha, 1}\|f\|_{\alpha} .
$$

Corollary 2.6. Let the conditions of Corollary 2.5 be satisfied. Then

$$
\left(|f|,\left|u_{z}\right|+\left|\nabla u_{z}\right|\right)_{0} \leqq C \varepsilon^{-(1-\alpha / \beta)}\|f\|_{\alpha}^{2} .
$$

\section{The Limiting Absorption and Amplitude Principles}

Lemma 3.1. Let the conditions (1.4), (1.7). (1.8), $f_{j} \in L_{2}^{2}, j=1,2$ be satisfied. Then for $\varepsilon \neq 0, a \leqq \lambda \leqq b, a>0$, the following inequality is valid:

$$
\left|\left(u_{z}^{(1)}, \bar{u}_{z}^{(2)}\right)\right| \leqq C \varepsilon^{-\delta}\left\|f_{1}\right\|_{2}\left\|f_{2}\right\|_{2},
$$

where $u_{z}^{(j)}=R_{z} f_{j}, z=\lambda+\varepsilon i, c, \delta$ do not depend on $f_{j}, \lambda, \varepsilon, 0 \leqq \delta<1$.

Proof. Without loss of generality we can assume that (2.10) is valid. Multiplying the equations

$$
\Delta u_{z}^{(j)}+z \mu u_{z}^{(j)}+\mu f_{j}=0, \quad j=1,2
$$

by $\rho \partial_{\rho} u_{z}^{(3-j)}$, where $\rho=1 \times 1$, and integrating over $\mathbb{R}^{n}$ we obtain

$$
\begin{aligned}
& (n-2) \int\left\langle\nabla u_{z}^{(1)}, \nabla \bar{u}_{z}^{(2)}\right\rangle d x-n z\left(u_{z}^{(1)}, \bar{u}_{z}^{(2)}\right) \\
& \quad+z\left(\mu_{2}-\mu_{1}\right) \int_{S}\langle x, v\rangle u_{z}^{(1)} u_{z}^{(2)} d x+\sum_{j=1}^{2}\left(\rho f_{j}, \partial_{\rho} \bar{u}_{z}^{(3-j)}\right)=0 .
\end{aligned}
$$

From (3.2)

$$
\int\left\langle\nabla u_{z}^{(1)}, \nabla \bar{u}_{z}^{(2)}\right\rangle d x=z\left(u_{z}^{(1)}, \bar{u}_{z}^{(2)}\right)+\left(f_{1}, \bar{u}_{z}^{(2)}\right) .
$$


Since (3.3), (3.4)

$$
\begin{aligned}
2\left(u_{z}^{(1)}, \bar{u}_{z}^{(2)}\right)= & z\left(\mu_{2}-\mu_{1}\right) \int_{S}\langle x, v\rangle u_{z}^{(1)} u_{z}^{(2)} d x \\
& +\sum_{j=1}^{2}\left(\rho f_{j}, \partial_{\rho} \bar{u}_{z}^{(3-j)}\right)+(n-2)\left(f_{1}, \bar{u}_{z}^{(2)}\right) .
\end{aligned}
$$

Because of (1.8), (2.14), $\lambda \in[a, b], 0<a<b$ we have

$$
\begin{aligned}
& \left|z\left(\mu_{2}-\mu_{1}\right) \int_{S}\langle x, v\rangle u_{z}^{(1)} u_{z}^{(2)} d x\right|^{2} \\
& \quad \leqq C\left(\left|f_{1}\right|,\left|u_{z}^{(1)}\right|+\left|\nabla u_{z}^{(1)}\right|\right)_{0}\left(\left|f_{2}\right|,\left|u_{z}^{(2)}\right|+\nabla u_{z}^{(2)} \mid\right)_{0} .
\end{aligned}
$$

Using (3.6), $f_{j} \in L_{2}^{2}$ and (2.36), where $\alpha=2$, we obtain

$$
\mid z\left(\mu_{2}-\mu_{1}\left|\int_{S}\langle x, v\rangle u_{z}^{(1)} u_{z}^{(2)} d x\right| \leqq C \varepsilon^{-\delta_{0}}\left\|f_{1}\right\|_{2}\left\|f_{2}\right\|_{2},\right.
$$

where $0 \leqq \delta_{0}<1$. Let us estimate the second term in (3.5):

$$
\left|\left(\rho f_{j}, \partial_{\rho} \bar{u}_{z}^{(3-j)}\right)\right| \leqq C\left\|f_{j}\right\|_{2}\left\|\nabla u_{z}^{(3-j)}\right\|_{0} .
$$

Since (2.33)

$$
\left\|\nabla u_{z}^{(3-j)}\right\|_{0}^{2} \leqq C \varepsilon^{-1}\left(\left|f_{3-j}\right|,\left|u_{z}^{(3-j)}\right|+\left|\nabla u_{z}^{(3-j)}\right|\right)_{0} .
$$

It now follows from (3.8), (3.9), (2.36) that

$$
\left|\left(\rho f_{j}, \partial_{\rho} \bar{u}_{z}^{(3-j)}\right)\right| \leqq C \varepsilon^{-\left(1+\delta_{0}\right) / 2}\left\|f_{1}\right\|_{2}\left\|f_{2}\right\|_{2} .
$$

In the same way

$$
\left|\left(f_{1}, \bar{u}_{z}^{(2)}\right)\right| \leqq C \varepsilon^{-\left(1+\delta_{0}\right) / 2}\left\|f_{1}\right\|_{0}\left\|f_{2}\right\|_{2} .
$$

Now (3.1) follows from (3.5), (3.7), (3.10), (3.11). The proof is complete.

Theorem 3.1. Let (1.4), (1.7), (1.8) be satisfied, $f \in L_{2}^{2}$. Then for $\varepsilon \neq 0,0<\alpha \leqq \lambda \leqq b$ the following inequality.

$$
\left\|\frac{d u_{z}}{d z}\right\|_{-2.2} \leqq C \varepsilon^{-\delta}\|f\|_{2}
$$

holds, where $0 \leqq \delta<1, c, \delta$ do not depend on $f, z$.

Proof. Because of the Hilbert identity for $R_{z}$ we have

Hence for any $g \in L_{2}^{2} \subset L^{2}(\mu)$.

$$
\frac{d R_{z}}{d z}=R_{z}^{2}
$$

$$
\left(\frac{d R_{z}}{d z} f, \bar{g}\right)=\left(R_{z} f, \overline{R_{z} g}\right) .
$$

With the help of Lemma 3.1

$$
\left|\left(\frac{d R_{z}}{d z} f, \bar{g}\right)\right| \leqq C \varepsilon^{-\delta}\|f\|_{2}\|g\|_{2} .
$$


Put here

$$
g=(1+|x|)^{-2} \frac{d R_{z}}{d z} f
$$

Then

$$
\left\|\frac{d R_{z}}{d z} f\right\|_{-2} \leqq C \varepsilon^{-\delta}\|f\|_{2} .
$$

Now (3.12) follows from the inequality (2.26) and the equation

$$
A v=z v+u_{z}
$$

where

$$
v=\frac{d u_{z}}{d z}
$$

The proof is complete.

The inequality (3.12) implies the validity of LABP for the operator $A$ :

Theorem 3.2. Let the conditions (1.4),(1.7),(1.8) be satisfied. Then for each $\lambda>0$ there exist the limits

$$
\lim _{\varepsilon \rightarrow \pm 0} R_{z}=R_{\lambda}^{ \pm}
$$

where $z=\lambda+\varepsilon i$ and the convergence takes place in the space $P$ of $L_{2}^{2} \rightarrow L_{-2}^{2}$-bounded operators. Moreover for $\varepsilon \in(0,1], \lambda \in[a, b], a>0$, the inequality

$$
\left\|R_{\lambda \pm \varepsilon i}-R_{\lambda}^{ \pm}\right\|_{P} \leqq C \varepsilon^{1-\delta}
$$

is valid, where $0 \leqq \delta<1, c, \delta$ do not depend on $\varepsilon, \lambda$. Further for any $\lambda_{1}, \lambda_{2}, a \leqq \lambda_{1}<$ $\lambda_{2} \leqq b$, where $a, b$ are arbitrary positive numbers, we have

$$
\left\|R_{\lambda_{2}}^{ \pm}-R_{\lambda_{1}}^{ \pm}\right\|_{P} \leqq C\left(\lambda_{2}-\lambda_{1}\right)^{\delta_{0}}
$$

where $0<\delta_{0} \leqq 1, c, \delta_{0}$ do not depend on $\lambda_{1}, \lambda_{2}$.

Thus the conditions i), ii) of Theorem 1.1 are satisfied for $B=L_{-2}^{2}, f \in L_{2}^{2}$. But the validity of iii) is obvious (see (2.25)) only for $B=L_{-\beta}^{2}, f \in L_{\beta}^{2}$, where $\beta$ is defined in Theorem 2.1. Because of Theorem 1.1 and Corollary 2.3 we obtain LAP in the following form:

Theorem 3.3. Let the conditions (1.4), (1.7), (1.8) be satisfied, $f \in L_{\beta}^{2}, f_{1} \in D(A) \cap L_{\beta}^{2}$, $f_{2} \in D(\sqrt{A}) \cap L_{\beta}^{2}$, where $\beta>3$ for $n=3$ and $\beta=3$ for $n>3, \omega>0$, and let $w$ be the solution of the problem (1.1), (2.28), (2.29). Then

$$
\lim _{t \rightarrow+\infty}\left(w-e^{-i \omega t} R_{\omega^{2}}^{+}\left(\mu^{-1} f\right)\right)=0,
$$

where the convergence takes place in the space $H_{-\beta}^{2}$.

Acknowledgments. This work was supported by SFB-123, Heidelberg University. I would like to express my gratitude to Professor Dr. W. Jäger for our fruitful discussions of the problem. 


\section{References}

1. Eidus, D.: The principle of limiting amplitude. Russ. Math. Surv. 24, No. 3, 97-167 (1969)

2. Eidus, D., Vinnik, A.: The principle of limiting amplitude for domains of the paraboloid type. Izv. Vyssh. Učhebn. Zaved. Mat. 3, 28-37 (1979)

3. Jäger, W., Rejto, P.: Limiting absorption principle for some Schrödinger operators with exploding potentials. J. Math. Anal. Appl. 91, 192-228, 95, 169-194 (1983)

4. Morawetz, C.: The limiting amplitude principle. Commun. Pure Appl. Math. 15, 349-361 (1962)

5. Saito, Y.: Schrödinger Operators with a non-spherical radiation condition. Preprint (1985)

Communicated by L. Nirenberg

Received January 3, 1986 